ARTICLE OPEN

\title{
Oxygen-vacancy induced magnetic phase transitions in multiferroic thin films
}

\author{
César Menéndez (D) ${ }^{1}$, Dewei $\mathrm{Chu}^{1}$ and Claudio Cazorla $\mathbb{D D}^{1 凶}$
}

Multiferroics in which giant ferroelectric polarization and magnetism coexist are of tremendous potential for engineering disruptive applications in information storage and energy conversion. Yet the functional properties of multiferroics are thought to be affected detrimentally by the presence of point defects, which may be abundant due to the volatile nature of some constituent atoms and the high temperatures involved in the synthesis of materials. Here, we demonstrate with theoretical methods that oxygen vacancies may enhance the functionality of multiferroics by radically changing their magnetic interactions in thin films. Specifically, oxygen vacancies may restore missing magnetic super-exchange interactions in large axial ratio phases, leading to full antiferromagnetic spin ordering, and induce the stabilization of ferrimagnetic states with considerable net magnetizations. Our theoretical study should help to clarify the origins of long-standing controversies in bismuth ferrite and improve the design of technological applications based on multiferroics.

npj Computational Materials (2020)6:76; https://doi.org/10.1038/s41524-020-0344-3

\section{INTRODUCTION}

Finding multiferroics in which ferroelectricity and magnetism coexist and influence each other is of great fundamental and applied interests ${ }^{1,2}$. Salient technological features of multiferroics include the control of the magnetization with electric fields to design efficient logic and memory devices ${ }^{3,4}$, and the realization of large piezomagnetic coefficients to facilitate the miniaturization of antennas and sensors ${ }^{5,6}$. Furthermore, competition between phases displaying distinct electric polarization and magnetic ordering offers also encouraging prospects for energy conversion applications like photovoltaics and solid-state cooling ${ }^{7-10}$.

But, unfortunately, multiferroics are rare in nature, typically present weak magnetoelectric coupling $\left(\mathrm{BiFeO}_{3}\right)^{11}$, and require extreme thermodynamic conditions for their synthesis $\left(\mathrm{PbVO}_{3}\right.$ and $\left.\mathrm{BiCoO}_{3}\right)^{12-14}$. In addition, magnetoelectric multiferroics mostly are antiferromagnetic hence potential applications based on external magnetic bias generally are frustrated due to their little influence on antiparallel magnetic spins ${ }^{15}$. Common strategies employed to synthesize bettered multiferroic materials include doping ${ }^{16,17}$, solid solutions $^{18,19}$, and strain engineering ${ }^{1,20}$.

Through epitaxial strain, it is actually possible to create new multiferroic materials in the laboratory that exhibit giant electric polarization and unexpected magnetic spin ordering ${ }^{21-23}$. An illustrative example is given by $\mathrm{BiFeO}_{3}$ (BFO) thin films, in which large spontaneous polarization and ferromagnetism (FM) have been observed under moderate compressive biaxial strains at room temperature 24,25 . The origins of the net magnetization reported for BFO thin films, however, are not clear yet and from a technological point of view it is crucial to understand them correctly.

Most magnetic ferroelectrics with chemical formula $\mathrm{AMO}_{3}$ and perovskite-like structure present antiferromagnetic spin ordering along the three pseudo-Cartesian directions (AFM-G), due to the dominant role of oxygen-mediated super-exchange interactions between neighbouring transition metal atoms $M^{26-29}$. In large axial ratio structures $(c / a>1)$, also known as "super-tetragonal", the covalency of $M-O$ bonds oriented along the electric polarization direction is significantly reduced and consequently the magnetic exchange interactions, which favour parallel magnetic spins, dominate in that direction. The coexistence of "in-plane" antiferromagnetism and "out-of-plane" ferromagnetism in super-tetragonal phases (AFM-C, Fig. 1a, b) leads anyway to null crystal magnetization when small spin canting effects are neglected. Therefore, intrinsic and robust FM in principle is not expected to occur in BFO or any other similar multiferroic ${ }^{30-33}$.

A plausible explanation for the appearance of $\mathrm{FM}$ in $\mathrm{AMO}_{3}$ perovskite oxide thin films is based on extrinsic causes like point defects ${ }^{25,34}$. The volatile nature of bismuth and the high temperatures involved in the preparation of samples, for instance, make the presence of oxygen vacancies $\left(V_{O}\right)$ almost inevitable in $\mathrm{Bi}$ based multiferroics ${ }^{17,19}$. In fact, oxygen defects may modify significantly the structural and functional properties of perovskite thin films via changes in the $M$ oxidation states and their coupling with the lattice strain ${ }^{35-40}$. However, a number of theoretical works based on first-principles methods have agreed in that the combined action of $V_{O}$ and lattice strain may not affect considerably the magnetic properties of Bi-based multiferroics ${ }^{41-43}$.

Here, we present new theoretical evidence showing that the presence of $V_{O}$ may in fact change radically the magnetic properties of multiferroic thin films via previously overlooked electro-structural mechanisms. We select $\mathrm{BiCoO}_{3}$ (BCO) as the model multiferroic in which to perform first-principles calculations based on density functional theory (DFT) because (i) this material already exhibits a large axial ratio in the absence of any strain, (ii) the magnetic effects that we predict could be realized on substrates that are commonly employed for growth of epitaxial perovskite thin films (Fig. 1C), and (iii) BCO naturally exhibits a high concentration of oxygen vacancies (i.e., it decomposes easily at temperatures around $700 \mathrm{~K}$ and needs to be synthesised at high pressures ${ }^{13,14}$ ). In particular, it is found that oxygen vacancies occupying specific lattice positions can induce the stabilization of full antiferromagnetic (AFM-G) super-tetragonal and ferrimagnetic (FiM) monoclinic polar phases, depending on the lattice strain. As a consequence, phase competition is enriched and magnetic

\footnotetext{
${ }^{1}$ School of Materials Science and Engineering, UNSW Sydney, Sydney, NSW 2052, Australia. ${ }^{\circledR}$ email: c.cazorla@unsw.edu.au
} 


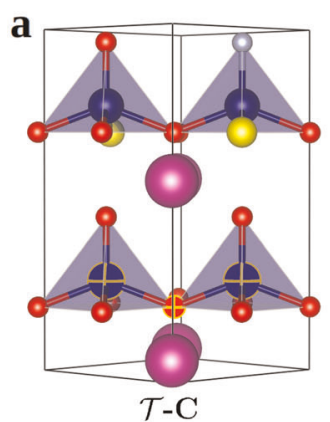

(Stoichiometric) $\circ$

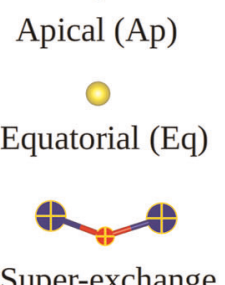

(SE) Fig. 1 Sketch of multiferroic $\mathrm{BiCoO}_{3}$ (BCO). a Representation of th
$\mathrm{BCO}$ and other multiferroics exhibiting giant electric polarization. $\mathbf{b}$
(AFM-C); green arrows represent atomic magnetic moments and their
displaying the effects predicted in this study (bottom); other we
comparison (top)
spheres, respectively.
functionalities further enhanced in comparison to perfectly
stoichiometric thin films. We show that most of the results obtained in BCO thin films can be generalized to BFO and other Bibased multiferroics, hence our conclusions are of broad applicability and significance to the field of multifunctional materials.

\section{RESULTS}

We have performed first-principles simulations of stoichiometric and non-stoichiometric BCO thin films based on density functional theory (DFT) techniques (see "Methods" section). The $V_{O}$-induced effects explained in this section have been observed for compositions $\mathrm{BiCoO}_{2.75}$ and $\mathrm{BiCoO}_{2.875}$. Detailed discussions on the influence of the selected DFT exchange-correlation energy functional and other technical parameters on our general conclusions can be found in the "Methods" and Supplementary Discussion sections. Overall, the results presented next are robust and qualitatively accurate.

$V_{O}$-induced effects on phase competition and functionality It is known both from experiments and first-principles calculations that bulk BCO presents a polar tetragonal $\mathcal{T}$ phase (space group $P 4 \mathrm{~mm}$ ) with a large axial ratio of $c / a \approx 1.3$ and relatively small lattice paramater $a=3.76 \AA^{9,14,31,44}$ (Fig. 1a). The competing structures are a non-polar orthorhombic $\mathcal{O}$ phase (space group Pnma) and a polar monoclinic $\mathcal{M}$ phase (space group PC)

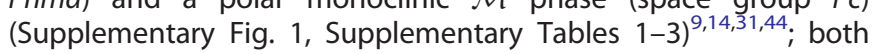
competing phases have cells that are slightly distorted versions of the ideal cubic perovskite structure with $c / a \approx 1$.

The polar phases in BCO present spontaneous polarizations along quite different crystallographic directions, namely, pseudocubic $[001]_{\mathrm{pc}}$ in $\mathcal{T}$ and $\sim[111]_{\mathrm{pc}}$ in $\mathcal{M}$. As regards magnetism, recent first- principles calculations have shown that phases $\mathcal{O}$ and $\mathcal{M}$ exhibit G-type antiferromagnetism (AFM-G) with a quite high Néel temperature, $T_{\mathrm{N}} \approx 500 \mathrm{~K}$, whereas the $\mathcal{T}$ phase presents $\mathrm{C}$ type antiferromagnetism (AFM-C) with a relatively low $T_{N}$ of $\approx$ $310 \mathrm{~K}^{31}$. In stoichiometric BCO thin films, and by completely neglecting temperature effects, a multiferroic $\mathcal{T} \rightarrow \mathcal{M}$ phase transition involving large structural, polar, and magnetic changes has been predicted to occur at in-plane parameter $a_{i n}=3.91 \AA^{9}$ (Fig. 2a-c).

Figure 2a-c show the influence of neutral oxygen vacancies (see "Methods" section), $V_{O}$, on the structural, ferroelectric, and magnetic properties of $\mathrm{BCO}$ thin films (the accompanying changes in atomic lattice positions and energy band gap are reported in Supplementary Tables 4-7 and Supplementary Fig. 2). For the smallest in-plane lattice parameters, a $\mathcal{T}$-C phase (magnetic spin

ordering is indicated along with the structure symmetry) containing oxygen vacancies in equatorial (Eq) positions (Fig. 1a) renders the lowest energy. The electric polarization and Néel temperature calculated for $\mathcal{T}-\mathrm{C}(\mathrm{Eq})$ are significantly lower than for the analogous stoichiometric phase, in particular, we estimate differences of $\Delta P \approx-75 \mu \mathrm{C} \mathrm{cm}^{-2}$ and $\Delta T_{\mathrm{N}} \approx-50 \mathrm{~K}$ when considering identical in-plane parameters.

At $a_{\text {in }}=3.77 \AA$, an unusual magnetic phase transition from AFM-C to AFM-G spin ordering occurs that is accompanied by the appearance of a small in-plane electric polarization $\left(P_{x y} \sim 10 \mu \mathrm{C}\right.$ $\mathrm{cm}^{-2}$ ) and change in $V_{O}$ symmetry. The Néel temperature of the $\mathcal{T}$-G(Ap) phase is lower than that of $\mathcal{T}-\mathrm{C}(\mathrm{Eq})$ by approximately $50 \mathrm{~K}$. Furthermore, at $a_{i n} \geq 3.91 \AA$ the system adopts a monoclinic ferrimagnetic (FiM) phase with oxygen vacancies preferably in equatorial positions, $\mathcal{M}$-FiM(Eq), and a considerable net magnetization (i.e., $0.5 \mu_{B}$ per formula unit for $\mathrm{BiCoO}_{2.75}$-Fig. $2 \mathrm{~d}$-and $0.25 \mu_{B}$ for $\left.\mathrm{BiCoO}_{2.875}\right)$. The Néel temperature of the $\mathcal{M}$-FiM(Eq) phase is larger than that of $\mathcal{T}-\mathrm{G}(\mathrm{Ap})$ and remains close to room temperature almost independently of $\mathrm{a}_{i n}$.

Interestingly, the formation energy of neutral oxygen vacancies in BCO thin films (see "Methods" section) is found to decrease steadily for $a_{i n} \geq 3.77 \AA$ as compared to the bulk equilibrium system (Supplementary Fig. 3). For instance, at $a_{\text {in }}=3.95 \AA$ the formation energy of the most stable type of $V_{O}$ is found to be $0.25 \mathrm{eV}$ smaller than obtained at $3.76 \AA$ (per formula unit). The difference in formation energy between $A p$ and Eq oxygen vacancies can be deduced directly from the energy curves shown in Fig. 2a (see "Methods" section). For instance, in the $\mathcal{M}$ phase the creation of an equatorial $V_{O}$ is $\sim 0.1 \mathrm{eV}$ more favourable than an apical vacancy, almost independently of the in-plane lattice parameter.

The physical mechanisms responsible for the two multiferroic phase transitions represented in Fig. $2 \mathrm{~d}$ will be explained in detail in the next subsections. Let us now comment briefly on the functionality enhancement deriving from the $\mathcal{T}-\mathrm{G}(\mathrm{Ap}) \rightarrow \mathcal{M}$-FiM (Eq) transformation. First, a large change in the electric polarization orientation involving a rotation of $\approx 60^{\circ}$ is observed during the transition (Fig. 2e); as a consequence, and in analogy to what has been observed in $\mathrm{Pb}\left(\mathrm{Zr}_{1-x} \mathrm{Ti}_{x}\right) \mathrm{O}_{3}$ alloys ${ }^{45,46}$ and $\mathrm{Bi}\left(\mathrm{Fe}_{1-x} \mathrm{Co}_{x}\right) \mathrm{O}_{3}$ thin films ${ }^{47}$, it should be possible to realize large piezoelectric responses under small electric bias for $a_{i n} \approx 3.91 \AA$. Second, the sizeable changes in electric polarization and total magnetization in principle should allow for control of the polarization with magnetic fields and vice versa, which hints at the likely existence of large magnetoelectric couplings ${ }^{1,2}$. And third, the thin film dimension along the direction perpendicular to the epitaxial plane shrinks by an impressive $\approx 11 \%$ (Fig. 2e) hence there is the 

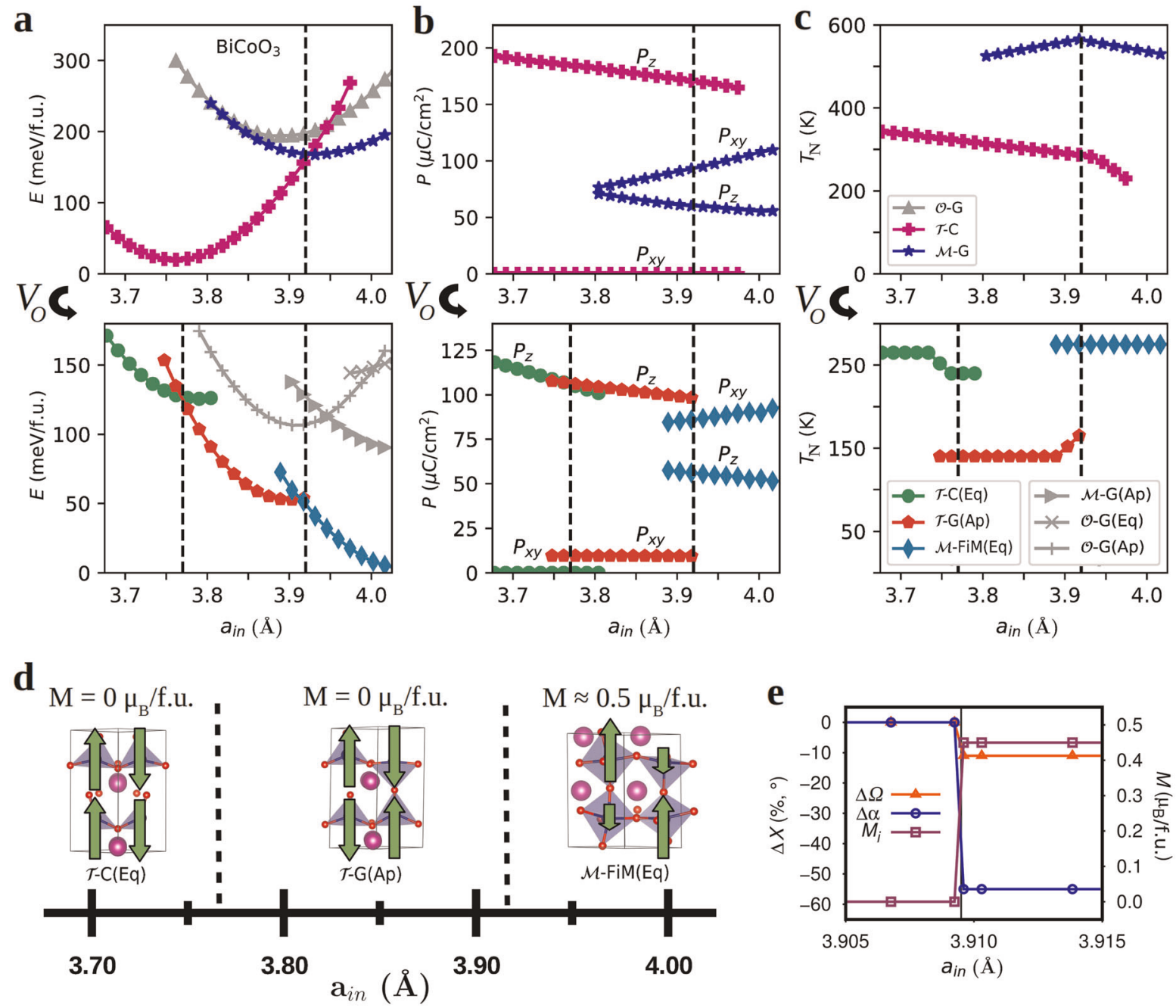

Fig. 2 Effects of oxygen vacancies on the phase competition and functionality of $\mathrm{BiCoO}_{3}$ (BCO) thin films. Results were obtained for composition $\mathrm{BiCoO}_{2.75}$. a Zero-temperature energy of competing phases expressed as a function of in-plane lattice parameter. Metastable phases are indicated by grey curves and strain-induced phase transitions by vertical dashed lines; the oxygen vacancy positions leading to the lowest energies, either apical "Ap" or equatorial "Eq", are indicated within parentheses. "G" stands for G-type antiferromagnetism, "C" for Ctype antiferromagnetism, and "FiM" for ferrimagnetism. b Electric polarization of stoichiometric and non-stoichiometric ground-state phases. c Magnetic transition temperature of stoichiometric and non-stoichiometric ground-state phases. $\mathbf{d}$ Phase transition sequence occurring in non-stoichiometric BCO thin films under increasing $a_{i n}$ i the green arrows represent the magnetic spin ordering in each phase. e Change in volume, $\Delta \Omega$, change in electric polarization orientation, $\Delta a$, and change in magnetic moment per formula unit, $M_{i}$, associated with the multiferroic $\mathcal{T}$-G $\rightarrow \mathcal{M}$-FiM phase transition.

possibility of realizing giant piezomagnetic responses ${ }^{5,6}$ and multicaloric effects ${ }^{8-10}$ through the application of external bias. As it will be shown later, similar magnetic phenomena than found for $\mathrm{BCO}$ are likely to occur also in other Bi-based multiferroic thin films, including $\mathrm{BiFeO}_{3}$.

$V_{O}$-induced magnetic super-exchange interactions in the $\mathcal{T}$ phase Figure 3 summarizes the electronic, structural, and magnetic properties of stoichiometric and non-stoichiometric $\mathcal{T}$ BCO thin films. In the stoichiometric $\mathcal{T}-C$ phase (Fig. 3a), the squarepyramidal $\mathrm{O}_{5}$ crystal field splits the electronic Co $d$ levels into nondegenerate $b_{2 g}\left(d_{x y}\right)$, doubly degenerate $e_{g}\left(d_{x z}, d_{y z}\right)$, and nondegenerate $a_{1 g}\left(d_{z^{2}}\right)$ and $b_{1 g}\left(d_{x^{2}-y^{2}}\right)$. Our first-principles calculations render a high-spin Co state characterised by the electronic occupation $b_{2 g}^{2} e_{g}^{2} a_{1 g}^{1} b_{1 g}^{1}$ and magnetic moment of $3.1 \mu_{B}$ (in absolute value), in good agreement with the available experimental data ${ }^{14}$.
In the non-stoichiometric $\mathcal{T}-C(E q)$ phase (Fig. 3b), the relative splitting of electronic $d$ levels remains invariant with respect to the stoichiometric case and the electronic occupation of the two cobalt ions nearest to the neutral $V_{O}$, which are reduced and are electronically equivalent (indicated with an orange plane in Fig. $3 \mathrm{~b})$, changes slightly to $b_{2 g}^{2} e_{g}^{3} a_{1 g}^{1} b_{1 g}^{1}$. Interestingly, when $V_{O}$ is created in an apical position the magnetic spin ordering of the $\mathcal{T}$ phase changes to AFM-G at specific $a_{i n}{ }^{\prime}$ s (Fig. 2a). Figure $3 c$ shows the electronic orbital occupation deduced from our DFT calculations for the two reduced cobalt ions in the $\mathcal{T}-G(A p)$ phase (indicated with an orange plane in Fig. $3 c$ ), which in this case turn out to be electronically inequivalent. In particular, the doubly degenerate $e_{g}\left(d_{x z}, d_{y z}\right)$ orbitals in the cobalt ion closest to the apical $V_{O}(\mathrm{Co} 3$, as labelled in Fig. 3d, e) undergo a significant energy reduction and become fully populated rendering the occupation state $e_{g}^{4} b_{2 g}^{1} a_{1 g}^{1} b_{1 g}^{1}$, while the other reduced metal ion (Co1, as labelled in Fig. $3 d$, e) exhibits the more usual distribution $b_{2 g}^{2} a_{1 g}^{2} e_{g}^{2} b_{1 g}^{1}$. 
a

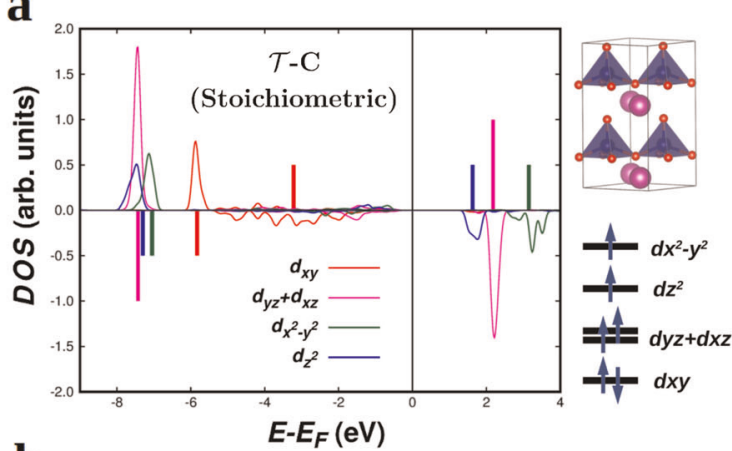

b

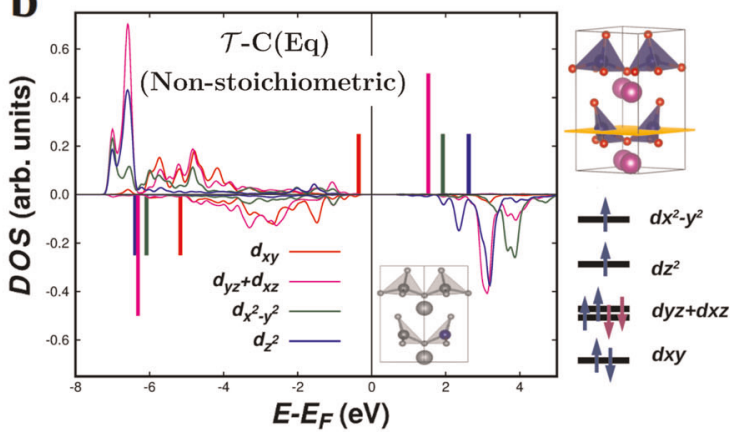

C
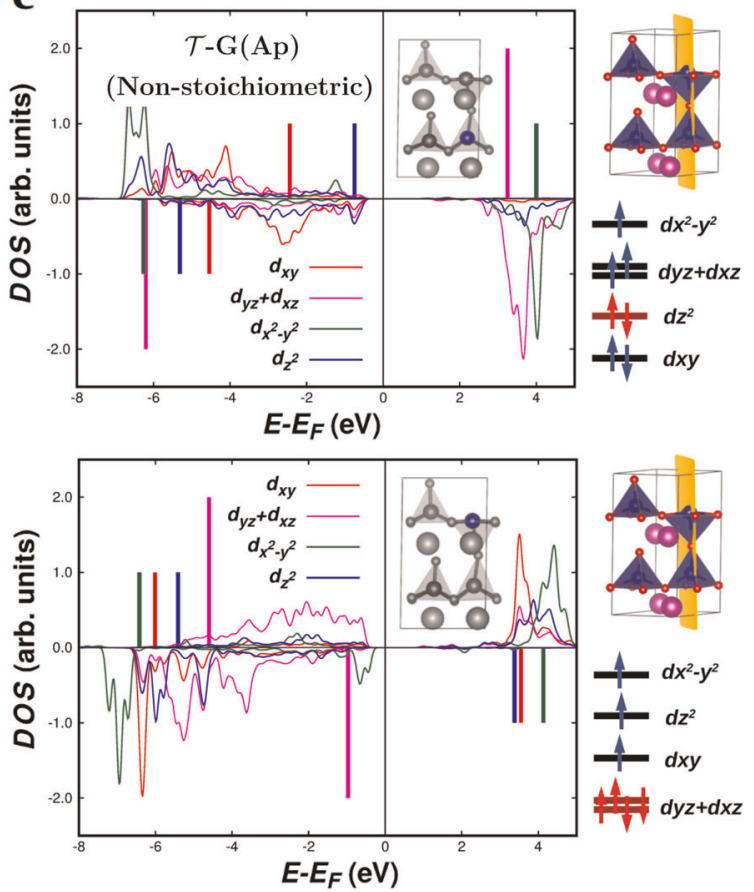

e

$\mathcal{T}$-G(Ap)

(Non-stoichiometric)
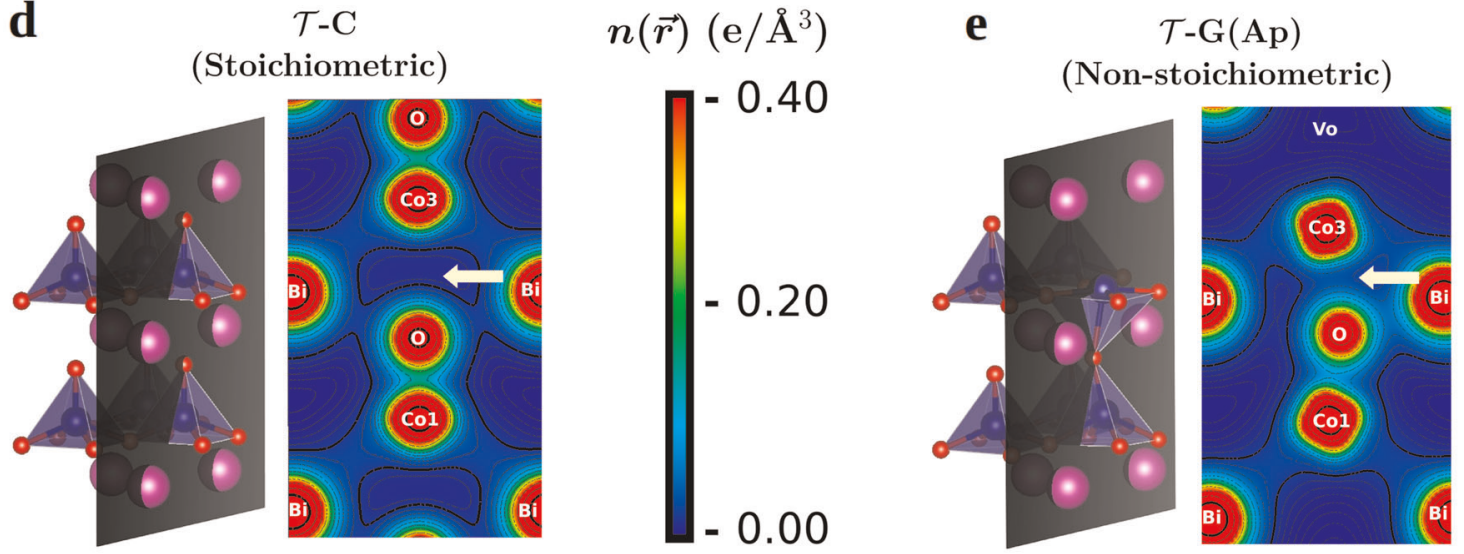

Fig. 3 Electronic, structural, and magnetic properties of $\mathcal{T} \mathbf{B i C o O}_{3}$ thin films. Results were obtained for composition $\mathrm{BiCoO}_{2.75}$ a Stoichiometric $\mathcal{T}$ phase. The first moment of the square of the $d$ densities of states (DOS) are indicated with thick vertical lines; from those moments the represented electronic orbital occupation sketch follows (idem for the following subfigures). Spin-up densities are represented in the top DOS panel and spin-down in the bottom DOS panel (idem for the following subfigures). b Non-stoichiometric $\mathcal{T}$ thin films with neutral oxygen vacancies $\left(V_{O}\right)$ in Eq positions and AFM-C spin ordering. The orange plane indicates the two Co ions that are reduced as a consequence of creating such $V_{O}$. The electronic orbital occupation sketch corresponds to the Co ion highlighted with blue colour in the transparency. The purple arrows in the $d$-orbitals occupation sketch indicate electronic delocalization. c Non-stoichiometric $\mathcal{T}$ thin films with neutral $V_{O}$ 's in Ap positions and AFM-G spin ordering. The orange plane indicates the two Co ions that are reduced as a consequence of creating such $V_{O}$. The electronic orbital occupations correspond to the Co ions highlighted with blue colour in the transparencies. The red arrows in the $d$-orbitals occupation sketch indicate differences with respect to the stoichiometric case. $\mathbf{d}$ Charge density surface plot for the stoichiometric $\mathcal{T}$ - $C$ phase; the surface over which the charge density is calculated is indicated by a grey plane in the structural ball-stick representation. Isovalue paths are represented with black and coloured lines. e Charge density surface plot for non-stoichiometric $\mathcal{T}$ - $\mathrm{G}(\mathrm{Ap})$. Regions of interest describing Co-O bonds are indicated with yellow arrows.

These drastic electronic rearrangements are correlated with the appearance of a strong structural distortion in the system that pushes $\mathrm{Co} 3$ towards the oxygen atom underneath of it (see inversion of the corresponding $\mathrm{O}_{5}$ square-pyramid in Fig. $3 \mathrm{c}$, e), which tends to restore (partially) the missing magnetic superexchange interactions along the out-of-plane direction. This superexchange restoration mechanism leading to the stabilization of AFM-G spin ordering, which is accompanied by a covalency increase in the out-of-plane $\mathrm{Co1-O-Co3} \mathrm{bonds,} \mathrm{is} \mathrm{clearly} \mathrm{observed}$ in the electronic density plots shown in Fig. $3 \mathrm{~d}$-e (see yellow arrows therein). We have explicitly checked that the stabilization of the $\mathcal{T}-G(A p)$ phase is robust with respect to the concentration of oxygen vacancies and presence of finite-size bias in our simulations (see "Methods" and Supplementary Discussion).

The $V_{O}$-induced AFM-C $\rightarrow$ AFM-G phase transition disclosed in large axial ratio BCO thin films may shed some light on uncomprehended experimental observations of antiferromagnetic spin ordering in other super-tetragonal multiferroic systems. For instance, first-principles works agree in predicting AFM-C spin ordering for $\mathcal{T}$ BFO thin films ${ }^{48-50}$ whereas in most experimental 
a

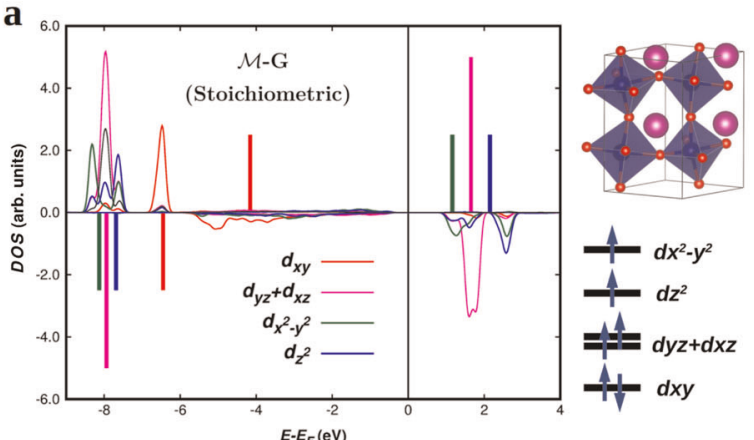

b

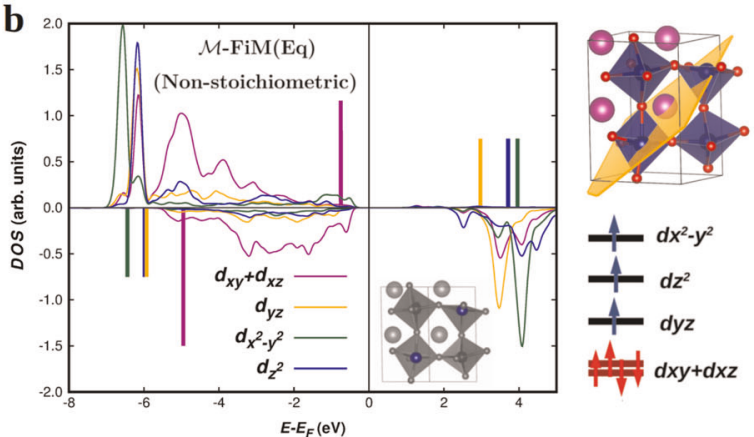

C $\mathrm{BiCoO}_{3}-\mathcal{M}-\mathrm{G}$

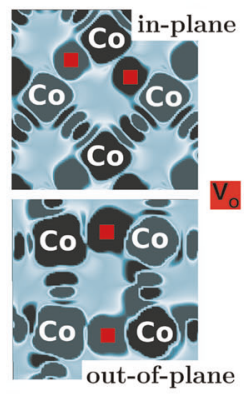

d

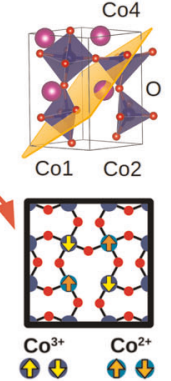

M-G

(Stoichiometric)

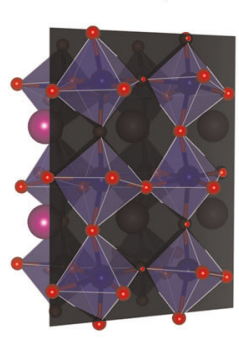

$n(\vec{r})\left(\mathrm{e} / \AA^{3}\right)$

e
M-FiM(Eq) (Non-stoichiometric)

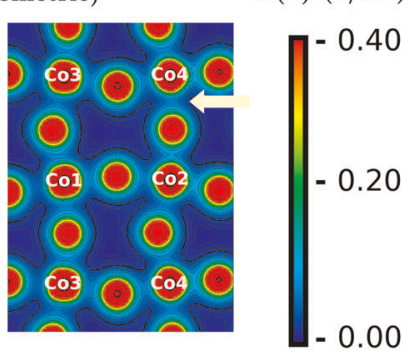

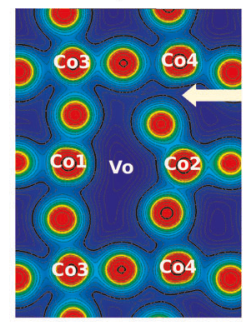

Fig. 4 Electronic, structural, and magnetic properties of $\mathrm{M} \mathrm{BiCoO}_{3}(\mathrm{BCO})$ thin films. Results were obtained for composition $\mathrm{BiCoO}$ 2.75. a Stoichiometric $\mathcal{M}-\mathrm{G}$ phase. The first moment of the square of the $d$ densities of states (DOS) are indicated with thick vertical lines; from those moments the represented electronic orbital occupation sketch follows (idem for the following subfigures). Spin-up densities are represented in the top DOS panel and spin-down in the bottom DOS panel (idem for the following subfigures). b Non-stoichiometric $\mathcal{M}$ thin films with neutral oxygen vacancies $\left(V_{O}\right)$ in Eq positions and FiM spin ordering. The orange plane indicates the two equivalent Co ions that are reduced as a consequence of creating such $V_{0}$. The electronic orbital occupation sketch corresponds to the Co ions highlighted with blue color in the transparency. The red arrows in the $d$-orbitals occupation sketch indicate the difference with respect to the stoichiometric case. c Spin-up (dark green) and spin-down (light green) electronic charge densities calculated for stoichiometric $\mathcal{M}$ BCO thin films considering two perpendicular planes; highly magnetized oxygen atoms are indicated with red squares. Stabilization of FiM spin ordering in the $\mathcal{M}$-FiM(Eq) phase is achieved when a highly magnetized equatorial oxygen atom is removed from the stoichiometric system. $\mathbf{d}$ Charge density surface plot for the stoichiometric $\mathcal{M}-\mathrm{G}$ phase; the surface over which the charge density is calculated is indicated by a grey plane in the structural ball-stick representation. Isovalue paths are represented with black and coloured lines. e Charge density surface plot for non-stoichiometric $\mathcal{M}$-FiM(Eq). Regions of interest describing structural distortions and Co-O bonds are indicated with yellow arrows.

studies AFM-G is reported for the same system ${ }^{51,52}$. As it will be shown later, by explicitly considering the presence of oxygen vacancies in BFO thin films such disagreements between theoretical and experimental works can be reconciled.

$V_{O}$-induced stabilization of a ferrimagnetic $\mathcal{M}$ phase

Figure 4 summarizes the electronic, structural, and magnetic properties of stoichiometric and non-stoichiometric $\mathcal{M}$ BCO thin films. In the stoichiometric $\mathcal{M}-\mathrm{G}$ phase (Fig. 4a), the octahedral $\mathrm{O}_{6}$ crystal field splits the electronic Co $d$ levels into doubly degenerate $e_{g}\left(d_{z^{2}}, d_{x^{2}-y^{2}}\right)$ and triply degenerate $t_{2 g}\left(d_{x y}, d_{y z}, d_{x z}\right)$. A strong Jahn-Teller distortion rendering a large $Q_{2}$ value of $0.37 \AA^{53}$ lifts further the degeneracy in the $e_{g}$ and $t_{2 g}$ manifolds ${ }^{54}$, promoting the electronic occupation state $b_{2 g}^{2} e_{g}^{2} a_{1 g}^{1} b_{1 g}^{1}$ (Fig. 4a).

Remarkably, when specific $V_{O}^{\prime}$ 's are created in equatorial positions the lowest-energy magnetic spin ordering becomes FiM. In particular, the net magnetization per formula unit associated with the $\mathcal{M}$-FiM(Eq) phase is considerable: 0.5 and $0.25 \mu_{B}$ for the off-stoichiometries $\mathrm{BiCoO}_{2.75}$ and $\mathrm{BiCoO}_{2.875}$, respectively. The two $\mathrm{Co}$ ions that are reduced by a neutral vacancy present the same magnetic moment orientation, equivalent electronic orbital occupancy, and sit within the $[111]_{p c}$ plane (indicated with an orange plane in Fig. 4b). Interestingly, the $\mathcal{M}$-FiM(Eq) phase appears only when highly magnetized oxygen atoms $\left(0.1-0.2 \mu_{B}\right)$ occupying equatorial positions in the stoichiometric $\mathcal{M}-\mathrm{G}$ crystal are removed (Fig. 4c).
For the pair of distant ions $\mathrm{Co} 1$ and $\mathrm{Co} 4$ to get reduced $\mathrm{CO}^{3+} \rightarrow$ $\mathrm{Co}^{2+}$, Fig. 4c), the crystal needs to undergo sizable structural distortions involving the $\mathrm{Bi}$ and $\mathrm{O}$ atoms that are close to the equatorial $V_{O}$ (Supplementary Tables 6-7). For example, the nonmagnetic oxygen in the apical position above $\mathrm{Co} 2$ is displaced significantly out of Co2-Co4 vertical (Fig. 4c). Such a concerted electro-structural process has a clear imprint on the electronic density calculated for the plane oriented perpendicular to the substrate that contains $\mathrm{Co} 1, \mathrm{Co} 2, \mathrm{Co}$, and $\mathrm{Co} 4$ (Fig. 4d, e, see yellow arrows therein). As can be appreciated in Fig. $4 d$, e, the covalency of the Co4-O bond in the $\mathcal{M}-\mathrm{FiM}(\mathrm{Eq})$ phase is reduced significantly as compared to that in the stoichiometric system. The vertical tilt of the $\mathrm{Co} 2-\mathrm{O}$ bond, which brings the apical oxygen closer to the equatorial $V_{O}$, is also evidenced in Fig. 4e. We have carried out several numerical tests to assess the influence of the dimensions of the simulation cell and concentration of oxygen vacancies on the findings just described. Our results indicate that the revealed $V_{O}$-induced stabilization of the $\mathcal{M}$ - $\mathrm{FiM}(\mathrm{Eq})$ phase is robust and general (see "Methods" and Supplementary Discussion).

The discovery of FiM spin ordering in the non-stoichiometric $\mathcal{M}$ phase motivated us to search for similar magnetic states, even if metastable, in the $\mathcal{T}$ and $\mathcal{O}$ BCO thin film geometries. The presence of highly magnetized $\mathrm{O}$ atoms was acknowledged in both stoichiometric phases (Supplementary Fig. 7), however, upon removal of those oxygens no net magnetization emerged (neglecting small spin canting effects). These results suggest that the structural distortions just described are key to the stabilization 


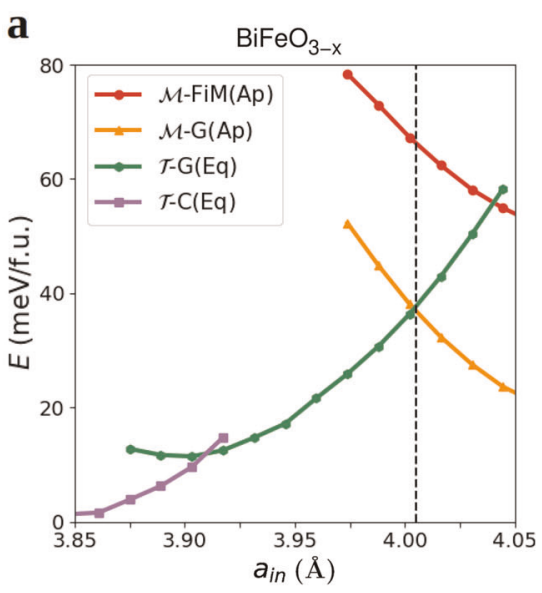

b
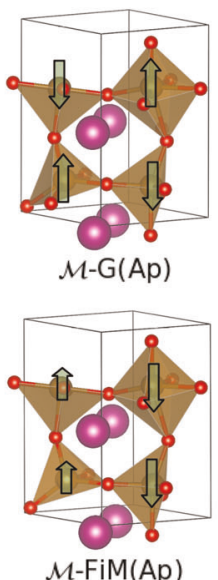

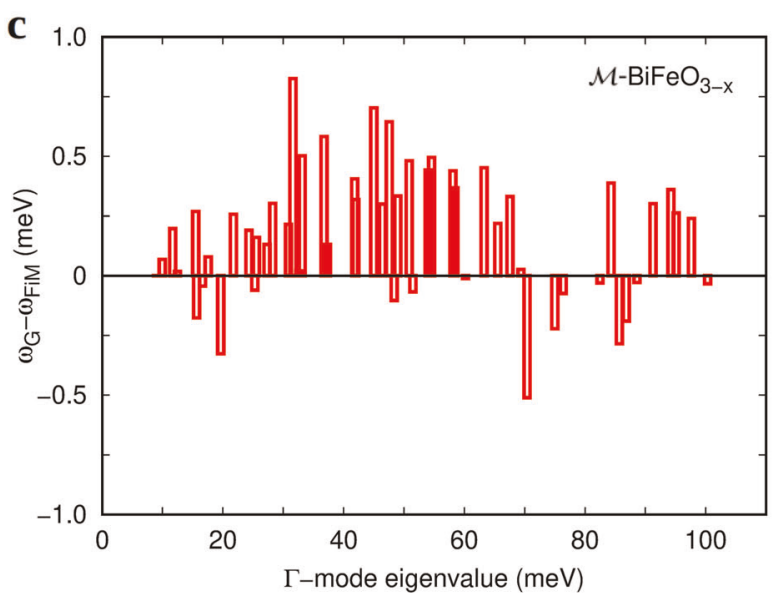

d $\mathrm{BiCoO}_{3}-\mathcal{M}-\mathrm{G}$
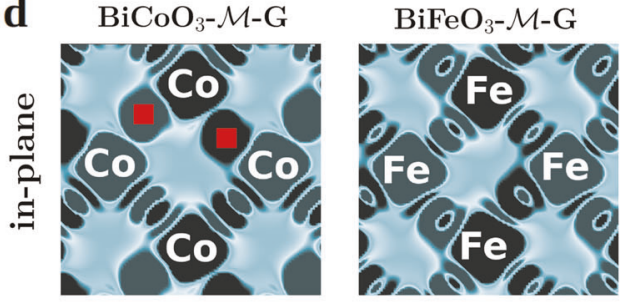

$\mathrm{BiFeO}_{3}-\mathcal{M}-\mathrm{C}$
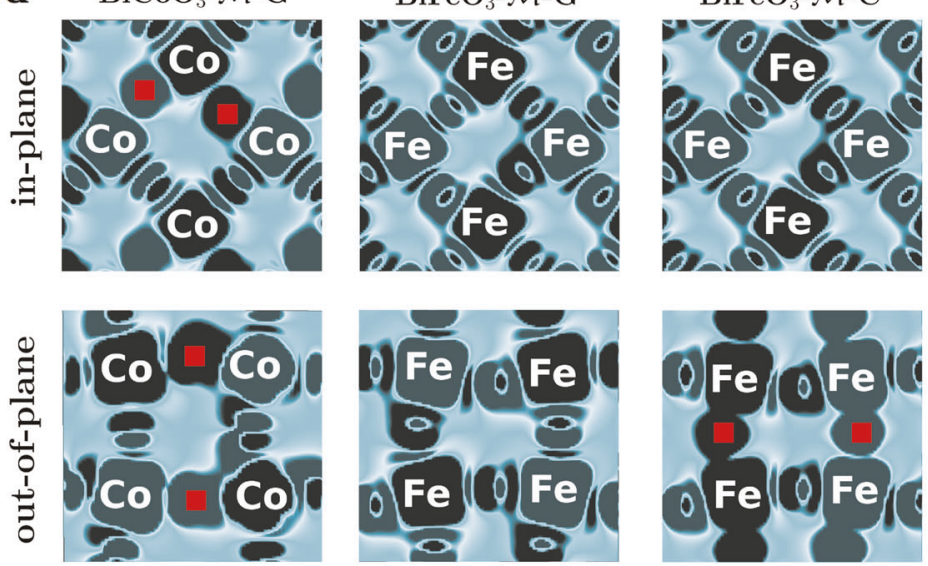

Fig. 5 Magnetic, structural, and vibrational properties of $\mathrm{BiFeO}_{3}$ (BFO) thin films containing neutral oxygen vacancies. a Zerotemperature energy of competing phases expressed as a function of in-plane lattice parameter. b Structural representation of nonstoichiometric $\mathcal{M}$ phases considering different magnetic orderings. $\mathbf{c} \Gamma$-point phonon spectrum calculated for non-stoichiometric monoclinic BFO thin films. For equivalent eigenmodes, the phonon frequencies of the $\mathcal{M}-\mathrm{G}(\mathrm{Ap})$ phase in general are higher than of the $\mathcal{M}$-FiM(Ap) phase hence the vibrational free energy of the latter phase is lower. $\mathbf{d}$ Spin-up (dark green) and spin-down (light green) electronic charge densities calculated for stoichiometric BCO and BFO thin films considering two perpendicular planes; highly magnetized oxygen atoms are indicated with red squares.

of FiM spin ordering. For instance, in the $\mathcal{T}$ phase the nonmagnetic oxygens appear in equatorial positions that are fairly clamped to the substrate (Supplementary Fig. 7), hence they hardly can move off-symmetry. Meanwhile, in the $\mathcal{O}$ phase the lack of polar order and high dielectric permittivity appears to impose high energy penalties to any symmetry-lowering distortion (Supplementary Table 7), thus frustrating FiM spin ordering.

Based on the previous outcomes, we hypothesize that the following three conditions may be necessary for the stabilization of $V_{O}$-induced $\mathrm{FiM}$ spin ordering in magnetic $\mathrm{AMO}_{3}$ oxide perovskites: (1) lack of inversion symmetry leading to polar order and structural deformation ease, (2) moderate axial ratio structures allowing for the reduction of second-nearest transition metal ions, and (3) the existence of highly magnetized oxygen ions.

\section{DISCUSSION}

The results obtained in $\mathrm{BCO}$ thin films raise the natural question: are there any other multiferroic materials in which similar $V_{O^{-}}$ induced magnetic phenomena may occur? To answer this question we investigated the special case of $\mathrm{BiFeO}_{3}$ (BFO) and other $\mathrm{Bi}$-based multiferroic $\left(\mathrm{BiMnO}_{3}\right.$ and $\left.\mathrm{BiCrO}_{3}\right)$ thin films. Figure 5 encloses the energy, magnetic, and vibrational properties of non-stoichiometric BFO thin films. In order to be consistent with the notation employed heretofore, we label the usual rhombohedral-like monoclinic phase of BFO as $\mathcal{M}$ and the large axial ratio tetragonal-like phase as $\mathcal{T}$ (in spite of the fact that the space groups corresponding to those structures are different in $\mathrm{BFO}$ and $\mathrm{BCO})^{50,55}$. Our zero-temperature calculations (Fig. 5a) predict a ground-state $\mathcal{M}-\mathrm{G}(\mathrm{Ap})$ phase at $a_{\text {in }} \geq 4.01 \AA$, followed by $\mathcal{T}-\mathrm{G}(\mathrm{Eq})$ at $3.91 \leq a_{\text {in }} \leq 4.01 \AA$, and $\mathcal{T}-\mathrm{C}(\mathrm{Eq})$ at $a_{\text {in }} \leq 3.91 \AA$. Indeed, when the likely existence of $V_{O}$ is explicitly considered in the simulations a broad $a_{i n}$ region appears in which AFM-G spin ordering is stable for the $\mathcal{T}$ phase. (We recall that AFM-G spin ordering is metastable in the corresponding stoichiometric system ${ }^{48,50}$ ). The causes of the stabilization of the $\mathcal{T}-\mathrm{G}(\mathrm{Eq})$ phase in BFO are very similar to those explained previously for $\mathcal{T}-\mathrm{G}(\mathrm{Ap})$ in BCO thin films (Fig. 3). As mentioned earlier, these results may shed new light on the origins of some unresolved discrepancies between theory and experiments regarding the identification of antiferromagnetic ordering in $\mathcal{T}$ BFO thin films ${ }^{48-52}$.

By creating an apical $V_{O}$ in the stoichiometric $\mathcal{M}-C$ phase, we found a thus far neglected ferrimagnetic phase in monoclinic BFO thin films, $\mathcal{M}$-FiM(Ap). In this case the net magnetization per formula unit amounts also to $0.5 \mu_{B}$ for $\mathrm{BiFeO}_{2.75}$. Nevertheless, the $\mathcal{M}$-FiM(Ap) phase is metastable at zero temperature due to a 
small energy difference of 25-30 meV per formula unit with respect to the ground-state phase $\mathcal{M}-\mathrm{G}(\mathrm{Ap})$ (Fig. 5a). The atomic structure of the metastable $\mathcal{M}-\mathrm{FiM}(\mathrm{Ap})$ and ground-state $\mathcal{M}-\mathrm{G}$ (Ap) phases are highly distorted and surprisingly very similar (Fig. 5b and Supplementary Tables 8-9). Analysis of the $\Gamma$-point phonon modes (Fig. 5c), however, indicates that the $\mathcal{M}$-FiM(Ap) phase is vibrationally softer than $\mathcal{M}-\mathrm{G}(\mathrm{Ap})$. Consequently, the $\mathcal{M}$-FiM(Ap) phase could be entropically stabilized over the $\mathcal{M}-\mathrm{G}$ (Ap) under increasing temperature due to the fact that the zerotemperature energies of the two states are similar and the vibrational free energy of the former phase appears to be more favourable ${ }^{9,31}$. Our results, therefore, can be interpreted as theoretical evidence showing that the observation of "ferromagnetic" behaviour in BFO thin films ${ }^{24}$ may be caused by the presence of oxygen vacancies (as it was suggested previously by other authors ${ }^{25}$ ).

Figure $5 d$ shows spin-up spin-down surface charge densities calculated for non-stoichiometric $\mathcal{M}$ and $\mathcal{T}$ BFO thin films considering in-plane and out-of-plane orientations. The reason why we could only find one FiM solution when generating neutral $V_{O}{ }^{\prime} s$ in stoichiometric $\mathcal{M}$ phases is now clear: all oxygen atoms in the $\mathcal{M}-\mathrm{G}$ phase are non-magnetic whereas all apical $\mathrm{O}$ in the $\mathcal{M}-\mathrm{C}$ phase are highly magnetized (Fig. $5 \mathrm{~d}$ ). The stoichiometric $\mathcal{T}$-C phase also displays highly magnetized oxygen atoms in apical positions however, as we have explained before, it seems difficult to observe FiM spin ordering in $c / a>1$ geometries. Furthermore, we repeated the same type of calculations and analysis for monoclinic-like $\mathrm{BiMnO}_{3}$ and $\mathrm{BiCrO}_{3}$ thin films, which fulfill conditions (1) and (2) above. We found that the appearance of FiM spin ordering is also correlated with the presence of highly magnetized oxygen atoms (Supplementary Fig. 8). In particular, a $\mathcal{M}$-FiM(Ap) phase displaying a net magnetization of $\approx 0.2 \mu_{B}$ is found in $\mathrm{BiCrO}_{2.75}$. These last results confirm that the simple rules provided in this work for understanding the appearance of FiM phases in non-stoichiometric multiferroic thin films may be general and robust.

In summary, by using first-principles calculations we have disclosed a number of previously overlooked electro-structural mechanisms induced by the presence of oxygen vacancies that facilitate the stabilization of unexpected magnetic states in multiferroic thin films. In particular, AFM-G and FiM spin orderings may naturally appear in large axial ratio and monoclinic phases under specific lattice strain conditions. Our theoretical results may clarify the origins of some long-standing controversies in BFO, the paradigm of single-phase multiferroics and one of the most intensively studied multifunctional materials. We provide general and simple rules to fundamentally understand and systematically predict FiM phases in non-stoichiometric multiferroics, thus offering new approaches for the rational engineering of bettered multifunctional materials. The present work shows that oxygen vacancies should be considered as a design opportunity to create new funcionalities in multiferroic thin films, especially to what concerns magnetism.

\section{METHODS}

Density functional theory calculations

First-principles spin-polarized calculations based on density functional theory (DFT) are performed with the generalized gradient approximation proposed by Perdew, Burke and Ernzerhof (GGA-PBE) as implemented in the VASP package ${ }^{56,57}$. We employ the "Hubbard-U" scheme derived by Dudarev et al. to deal with the $3 d$ electrons in $\mathrm{Co}(\mathrm{Fe})$ atoms and, as done in previous works, a $U$ value of 6 (4) eV is adopted ${ }^{9,30,31}$. We use the "projected augmented wave" method ${ }^{58}$ considering the following electronic states as valence: Co's $4 s^{1} 3 d^{8}$, Fe's $3 p^{6} 4 s^{1} 3 d^{7}, \mathrm{Mn}^{\prime} s 4 s^{1} 3 d^{6}, \mathrm{Cr}^{\prime} \mathrm{s} 4 s^{1} 3 d^{5}$, Bi's $6 s^{2} 5 d^{10} 6 p^{3}$, and O's $2 s^{2} 2 p^{4}$. The energy cut-off is truncated at $650 \mathrm{eV}$ and we employ a $\Gamma$-centered k-point grid of $4 \times 6 \times 6$ for a $2 \times \sqrt{2} \times \sqrt{2}$ supercell containing 20 atoms (that is, four formula units) ${ }^{55}$. The reported net magnetizations correspond to sums over the local magnetic moments, which are accurate to within $0.01 \mu_{B}$ per formula unit.

Thin film geometry relaxations are carried out by using a conjugated gradient algorithm that allows to change the simulation-cell volume and atomic positions while constraining the length and orientation of the two in-plane lattice vectors (that is, $|a|=|b|$ and $\gamma=90^{\circ}$ ). Periodic boundary conditions are applied along the three lattice-vector directions, thus the influence of surface and interface effects are systematically disregarded in our simulations. This type of calculations are known as "strained-bulk" geometry relaxations and typically are considered to be a good approximation for thin films with thicknesses of at least few nanometers $^{48,55}$ (i.e., for which surface and interface effects can be safely neglected). In our simulations, we assume systems that are elastically coupled to a substrate hence we disregard the existence of possible stress relaxation mechanisms in thin films. The geometry relaxations are stopped when the forces on the ions are smaller than $0.01 \mathrm{eV} / \AA$. We have checked the vibrational stability of every phase by estimating the lattice phonons at the $\Gamma$-point with the small-displacement method ${ }^{59}$ and considering central differences for the calculation of atomic forces derivatives. Full phonon spectra calculations have not been pursued in this work due to the huge computational expense associated with large and low-symmetry offstoichiometric unit cells.

In this study, most non-stoichiometric configurations are generated by removing one oxygen atom from an apical or equatorial position in a 20atoms simulation cell, thus rendering the chemical composition $\mathrm{BiCoO}_{2.75}$. Apical and equatorial $V_{O}$ positions are investigated systematically by generating all inequivalent configurations in all competing phases and considering all possible magnetic spin orderings (FM, AFM-G, AFM-G, and AFM- ${ }^{31}$ ). Additional simulations in a 40 -atoms simulation cell have been performed to assess the influence of size effects and $V_{O}$ concentration in our main conclusions (Supplementary Fig. 10 and Supplementary Discussion). The results of our tests indicate that our findings obtained for $\mathrm{BiCoO}_{2.75}$ are general and qualitatively correct also for $\mathrm{BiCoO}_{2.875}$ thin films. The influence of possible kinetic effects on the distribution of oxygen vacancies has been totally neglected in this study.

The results presented in the main text apply to neutral oxygen vacancies since we have found that the creation of neutral $V_{O}$ 's in energetically more favourable than that of charged oxygen vacancies (Supplementary Fig. 9, Supplementary Table 10, and Supplementary Discussion). In particular, to estimate the ranking of $V_{O}$ formation energies as a function of charge, $q$, we have employed the following well-established formula ${ }^{60}$ :

$E_{\text {def }}\left[V_{O}^{q}\right]=E\left[V_{O}^{q}\right]-E_{\text {stoi }}+E_{\text {corr }}^{q}-n_{O} \mu_{O}+q\left[\epsilon_{F}+\epsilon_{V}+\Delta V\right]$,

where $E\left[V_{O}^{q}\right]$ is the energy of the non-stoichiometric system containing the oxygen vacancy, $E_{\text {stoi }}$ the energy of the corresponding stoichiometric system, $E_{\text {corr }}^{q}$ a finite-size supercell correction, $n_{O}$ the number of created $V_{O}$ 's (typically equal to 1 in our calculations), $\mu_{O}$ the chemical potential of oxygen atoms, $\epsilon_{F}$ the Fermi energy in the non-stoichiometric system, $\epsilon_{V}$ the top energy in the valence band of the non-stoichiometric system, and $\Delta V$ a term used for aligning the electrostatic potentials of the stoichiometric and defective supercells. In order to calculate $E_{\text {corr }}^{q}$ and $\Delta V$, we have followed the methods explained in work ${ }^{60}$. According to our estimations, neutral $V_{O}^{\prime}$ s $(q=0)$ are energetically more favourable than charged vacancies $(q=$ $+2 e$ ) by about $\sim 1 \mathrm{eV}$ per formula unit (Supplementary Fig. 9, Supplementary Table 10, and Supplementary Discussion).

We have performed several tests to assess the influence of the adopted DFT exchange-correlation functional and $U$ value on our theoretical predictions (Supplementary Figs. 4-6, Supplementary Tables 11-13, and Supplementary Discussion). Specifically, we repeated most calculations by considering the PBEsol functional ${ }^{61}$ and $2 \leq U \leq 6 \mathrm{eV}$ values. It is found that the main conclusions presented in the main text are not affected qualitatively by the choice of the $U$ parameter or exchange-correlation functional. At the quantitative level, the $a_{\text {in }}$ parameters at which the phase transitions occur and the energy ranking of the competing phases change in some cases (Supplementary Figs. 4-6, Supplementary Tables 11-13, and Supplementary Discussion). For a detailed discussion on these technical aspects, see Supplementary Discussion. It is also worth noting that lattice thermal excitations have been neglected systematically throughout this study ${ }^{35}$. Nevertheless, we have performed a test to assess the influence of this approximation in our main findings (Supplementary Fig. 11 and Supplementary Discussion) and found that the conclusions reported in this study appear to be robust and physically meaningful also for $T \neq 0$ conditions.

Regarding the estimation of the electric polarization, $P$, we started by employing the Berry phase formalism ${ }^{62}$. However, the presence of oxygen 
vacancies induces a notable reduction in the energy band gap of the system (Supplementary Fig. 2) that in some cases frustrates the determination of the corresponding Berry phase (due to the appearance of intermediate metallic phases). In order to overcome such a limitation, we opted for calculating the electric polarization perturbatively. Specifically, we estimate $P$ with the formula ${ }^{55}$ :

$$
P_{\alpha}=\frac{1}{\Omega} \sum_{\kappa \beta} Z_{\kappa \beta a}^{*} u_{\kappa \beta},
$$

where $\Omega$ is the volume of the cell, $k$ runs over all the atoms, $a, \beta=x, y, z$ represent Cartesian directions, $\mathbf{u}_{k}$ is the displacement vector of the $k$-th atom as referred to a non-polar reference phase, and $\boldsymbol{Z}_{\boldsymbol{k}}^{*}$ the Born effective charge tensor calculated in a non-polar reference state $\left(\boldsymbol{Z}_{K}^{*}\right.$ has been calculated for each defective system). In the stoichiometric phases we do not find the technical limitations just explained for non-stoichiometric systems, hence in that case we have been able to compare the $P$ values obtained with the Berry phase approach (exact) and Eq. (2) (approximate). According to our estimations, the electric polarizations calculated perturbatively are accurate to within $\sim 10 \%$ of, and systematically larger than, the $P$ values calculated with the Berry phase method. It is reasonable to assume then a similar level of accuracy for the $P$ values estimated for non-stoichiometric thin films that are reported in Fig. 2.

\section{Heisenberg model Monte Carlo simulations}

To simulate the effects of thermal excitations on the magnetic ordering of $\mathcal{T}, \mathcal{O}$, and $\mathcal{M} B C O$ thin films, both stoichiometric and non-stoichiometric, we construct several spin Heisenberg models of the form $\hat{H}=\frac{1}{2} \sum_{i j} J_{i j} S_{i} S_{j}$, in which the value of the involved exchange constants are obtained from zero-temperature DFT calculations (following analogous methods to those explained in detail in previous works of ours ${ }^{9,30,31}$ ).

For the $\mathcal{T}$ phase $(c>a)$, we consider spin couplings between first nearest in-plane and out-of-plane neighbours ( $J_{a}$ and $J_{c}$, respectively) and between second nearest neighbours $\left(J_{a c}\right)$. The value of such magnetic exchange constants are obtained with the formulas ${ }^{9,30,31}$ :

$J_{a}^{\mathcal{T}}=\frac{1}{8|S|^{2}}\left(E_{\mathrm{eq}}^{\mathrm{FM}}+E_{\mathrm{eq}}^{\mathrm{A}}-E_{\mathrm{eq}}^{\mathrm{C}}-E_{\mathrm{eq}}^{\mathrm{G}}\right)$

$J_{c}^{\mathcal{T}}=\frac{1}{4|S|^{2}}\left(E_{\mathrm{eq}}^{\mathrm{FM}}-E_{\mathrm{eq}}^{\mathrm{A}}+E_{\mathrm{eq}}^{\mathrm{C}}-E_{\mathrm{eq}}^{\mathrm{G}}\right)$,

$J_{\text {ac }}^{\mathcal{T}}=\frac{1}{16|S|^{2}}\left(E_{\mathrm{eq}}^{\mathrm{FM}}-E_{\mathrm{eq}}^{\mathrm{A}}-E_{\mathrm{eq}}^{\mathrm{C}}+E_{\mathrm{eq}}^{\mathrm{G}}\right)$,

where $E$ represents energy and superscripts "FM", "G", " $A$ ", and " $\mathrm{C}$ " ferromagnetic, antiferromagnetic G-type, antiferromagnetic A-type, and antiferromagnetic $\mathrm{C}$-type spin arrangements, respectively. For the $\mathcal{O}$ and $\mathcal{M}$ phases $(c \approx a)$, we consider only spin couplings between first nearest neighbours and obtain their value with the expression ${ }^{9,30,31}$ :

$J_{a}^{\mathcal{O}, \mathcal{M}}=\frac{1}{6|S|^{2}}\left(E_{\text {eq }}^{\mathrm{FM}}-E_{\text {eq }}^{\mathrm{G}}\right)$.

The value of some characteristic magnetic exchange constants estimated in the manner just described are reported in Supplementary Table 14.

We use the Heisenberg models described above to perform Monte Carlo (MC) simulations in a periodically-repeated simulation box of $20 \times 20 \times$ 20 spins; thermal averages are computed from runs of 50,000 MC sweeps after equilibration. These simulations allow us to monitor the $T$ dependence of the magnetic ordering through the computation of the AFM-C (in the $\mathcal{T}$ phase) and AFM-G (in the $\mathcal{O}$ and $\mathcal{M}$ phases) order parameters, namely, $S^{\complement} \equiv \frac{1}{N} \sum_{i}(-1)^{n_{i x}+n_{i y}} S_{i z}$ and $S^{G} \equiv \frac{1}{N} \sum_{i}(-1)^{n_{i x}+n_{i y}+n_{i z}} S_{i z}$. Here, $n_{i x}, n_{i y}$ and $n_{i z}$ are the three integers locating the $i$-th lattice cell, and $N$ is the total number of spins in the simulation box. For the calculation of $S^{C}$ and $S^{G}$, we considered only the $z$ component of the spins because a small symmetry-breaking magnetic anisotropy was introduced in the Hamiltonian in order to facilitate the numerical analysis ${ }^{9,30,31}$.

\section{DATA AVAILABILITY}

The data that support the findings of this study are available from the corresponding author (C.C.) upon reasonable request.
Received: 3 July 2019; Accepted: 20 May 2020;

Published online: 05 June 2020

\section{REFERENCES}

1. Ramesh, R. \& Spaldin, N. A. Multiferroics: progress and prospects in thin films. Nat. Mater. 6, 21 (2007).

2. Spaldin, N. A. \& Ramesh, R. Advances in magnetoelectric multiferroics. Nat. Mater. 18, 203 (2019).

3. Heron, J. T. et al. Deterministic switching of ferromagnetism at room temperature using an electric field. Nature 516, 370 (2014).

4. Allibe, J. et al. Room temperature electrical manipulation of giant magnetoresistance in spin valves exchange-biased with $\mathrm{BiFeO}_{3}$. Nano Lett. 12, 1141 (2012).

5. Domann, J. P. \& Carman, G. P. Strain powered antennas. J. Appl. Phys. 121, 044905 (2017).

6. Nan, T. et al. Acoustically actuated ultra-compact NEMS magnetoelectric antennas. Nat. Commun. 8, 296 (2017).

7. Huang, W. et al. Multiferroic $\mathrm{Bi}_{2} \mathrm{FeCrO}_{6}$ based $\mathrm{p}$-i-n heterojunction photovoltaic devices. J. Mater. Chem. A 5, 10355 (2017).

8. Stern-Taulats, E. et al. Multicaloric materials and effects. MRS Bull. 43, 295 (2018).

9. Cazorla, C. \& Íñiguez, J. Giant direct and inverse electrocaloric effects in multiferroic thin films. Phys. Rev. B 98, 174105 (2018).

10. Cazorla, C. Novel mechanocaloric materials for solid-state cooling applications. Appl. Phys. Rev. 6, 041316 (2019).

11. Bertinshaw, J. et al. Direct evidence for the spin cycloid in strained nanoscale bismuth ferrite thin films. Nat. Commun. 7, 12664 (2016).

12. Belik, A. A., Azuma, M., Saito, T., Shimakawa, Y. \& Takano, M. Crystallographic features and tetragonal phase stability of $\mathrm{PbVO}_{3}$, a new member of $\mathrm{PbTiO}_{3}$ family. Chem. Mater. 17, 269 (2005).

13. Belik, A. A. et al. Neutron powder diffraction study on the crystal and magnetic structures of $\mathrm{BiCoO}_{3}$. Chem. Mater. 18, 798 (2006).

14. Oka, K. et al. Pressure-induced spin-state transition in $\mathrm{BiCoO}_{3}$. J. Am. Chem. Soc. 132, 9438 (2010).

15. Wang, X. et al. E-field control of the RKKY interaction in FeCoB/Ru/FeCoB/PMN-PT (011) multiferroic heterostructures. Adv. Mater. 30, 1803612 (2018).

16. Das, R. \& Mandal, K. Magnetic, ferroelectric, and magnetoelectric properties of Badoped $\mathrm{BiFeO}_{3}$. J. Magn. Magn. Mater. 324, 1913 (2012).

17. Das, R., Sharma, S. \& Mandal, K. Aliovalent $\mathrm{Ba}^{2+}$ doping: A way to reduce oxygen vancancy in multiferroic $\mathrm{BiFeO}_{3}$. J. Magn. Magn. Mater. 401, 129 (2016).

18. Sakai, $\mathrm{H}$. et al. Displacement-type ferroelectricity with off-center magnetic ions in perovskite $\mathrm{Sr}_{1-x} \mathrm{Ba}_{x} \mathrm{MnO}_{3}$. Phys. Rev. Lett. 107, 137601 (2011).

19. Hojo, H. et al. Development of bismuth ferrite as a piezoelectric and multiferroic material by cobalt substitution. Adv. Mater. 30, 1705665 (2018).

20. Martin, L. W. et al. Multiferroics and magnetoelectrics: thin films and nanostructures. J. Phys.: Condens. Matter 20, 434220 (2008).

21. Zhang, L. et al. Giant polarization in super-tetragonal thin films through interphase strain. Science 361, 494 (2018)

22. Varga, $\mathrm{T}$. et al. Coupled lattice polarization and ferromagnetism in multiferroic $\mathrm{NiTiO}_{3}$ thin films. ACS Appl. Mater. Interfaces 9, 21879 (2017).

23. Töpfer, J. \& Goodenough, J. G. LaMnO ${ }_{3+\delta}$ revisited. J. Solid State Chem. 130, 117 (1997).

24. Wang, J. et al. Epitaxial $\mathrm{BiFeO}_{3}$ multiferroic thin film heterostructures. Science 299, 1719 (2003)

25. Eerenstein, W. et al. Comment on "Epitaxial $\mathrm{BiFeO}_{3}$ multiferroic thin film heterostructures". Science 307, 1203 (2005).

26. Goodenough, J. B. Theory of the role of covalence in the perovskite-type manganites (La, M (II)) $\mathrm{MnO}_{3}$. Phys. Rev. 100, 564 (1955).

27. Hill, N. A. \& Filipetti, A. Why are there any magnetic ferroelectrics? J. Magn. Magn. Mater. 242, 976 (2002).

28. Spaldin, N.A. Magnetic Materials: Fundamentals and Applications. 2nd edn (Cambridge University Press), https://doi.org/10.1017/CBO9780511781599.021 (2012).

29. Bhattacharya, A. \& May, S. Magnetic oxide heterostructures. Annu. Rev. Mater. Res. 44, 65 (2014).

30. Cazorla, C. \& Íñiguez, J. Insights into the phase diagram of bismuth ferrite from quasiharmonic free-energy calculations. Phys. Rev. B 88, 214430 (2013).

31. Cazorla, C., Diéguez, O. \& Íñiguez, J. Multiple structural transitions driven by spinphonon couplings in a perovskite oxide. Sci. Adv. 3, e1700288 (2017).

32. Singh, D. J. Electronic structure and bond competition in the polar magnet $\mathrm{PbVO}_{3}$. Phys. Rev. B 73, 094102 (2006).

33. Solovyev, I. V. Magnetic structure of the noncentrosymmetric perovskites $\mathrm{PbVO}_{3}$ and $\mathrm{BiCoO}_{3}$ : theoretical analysis. Phys. Rev. B 85, 054420 (2012).

34. Niu, W. et al. Direct demonstration of the emergent magnetism resulting from the multivalence $\mathrm{Mn}$ in a $\mathrm{LaMnO}_{3}$ epitaxial thin film system. Adv. Electron. Mater. 4, 1800055 (2018) 
35. Cazorla, C. Lattice effects on the formation of oxygen vacancies in perovskite thin films. Phys. Rev. Appl. 7, 044025 (2017).

36. Herklotz, A. et al. Strain coupling of oxygen non-stoichiometry in perovskite thin films. J. Phys.: Condens. Matter 29, 493001 (2017).

37. Mangalam, R. V. K., Ray, N., Waghmare, U. V., Sundaresana, A. \& Rao, C. N. R. Multiferroic properties of nanocrystalline $\mathrm{BaTiO}_{3}$. Solid State Commun. 149, 1 (2009).

38. Shimada, T. et al. Multiferroic vacancies at ferroelectric $\mathrm{PbTiO}_{3}$ surfaces. Phys. Rev. Lett. 115, 107202 (2015).

39. Shimada, T. et al. Multiferroic nature of intrinsic point defects in $\mathrm{BiFeO}_{3}$ : a hybrid Hartree-Fock density functional study. Phys. Rev. B 93, 174107 (2016).

40. Li, W. et al. Oxygen-vacancy-induced antiferromagnetism to ferromagnetism transformation in $\mathrm{Eu}_{0.5} \mathrm{Ba}_{0.5} \mathrm{TiO}_{3-\delta}$ multiferroic thin films. Sci. Rep. 3, 2618 (2013).

41. Ederer, C. \& Spaldin, N. A. Influence of strain and oxygen vacancies on the magnetoelectric properties of multiferroic bismuth ferrite. Phys. Rev. B 71, 224103 (2005).

42. Paudel, T. R., Jaswal, S. S. \& Tsymbal, E. Y. Intrinsic defects in multiferroic $\mathrm{BiFeO}_{3}$ and their effect on magnetism. Phys. Rev. B 85, 104409 (2012).

43. Chen, X.-Y. et al. Tuning the polarization and magnetism in $\mathrm{BiCoO}_{3}$ by strain an oxygen vacancy effect: a first-principle study. J. Appl. Phys. 111, 013901 (2012).

44. Diéguez, O. \& Íñiguez, J. First-principles investigation of morphotropic transitions and phase-change functional responses in $\mathrm{BiFeO}_{3}-\mathrm{BiCoO}_{3}$ multiferroic solid solutions. Phys. Rev. Lett. 107, 057601 (2011).

45. Jaffe, B., Roth, R. S. \& Marzullo, S. Piezoelectric properties of lead zirconate-lead titanate solid-solution ceramics. J. Appl. Phys. 25, 809 (1954).

46. Bellaiche, L., García, A. \& Vanderbilt, D. Finite-temperature properties of $\mathrm{Pb}\left(\mathrm{Zr}_{1-x}\right.$ $\left.\mathrm{Ti}_{x}\right)_{3}$ alloys from first principles. Phys. Rev. Lett. 84, 5427 (2000).

47. Shimizu, K., Hojo, H., Ikuhara, Y. \& Azuma, M. Enhanced piezoelectric response due to polarization rotation in cobalt-substituted $\mathrm{BiFeO}_{3}$ epitaxial thin films. Adv. Mater. 28, 8639 (2016).

48. Hatt, A. J., Spaldin, N. A. \& Ederer, C. Strain-induced isosymmetric phase transition in $\mathrm{BiFeO}_{3}$. Phys. Rev. B 81, 054109 (2010).

49. Diéguez, O., González-Vázquez, O. E., Wojdel, J. C. \& Îñiguez, J. First-principles predictions of low-energy phases of multiferroic $\mathrm{BiFeO}_{3}$. Phys. Rev. B 83, 094105 (2011).

50. Heo, Y. et al. Impact of isovalent and aliovalent doping on mechanical properties of mixed phase $\mathrm{BiFeO}_{3}$. ACS Nano 11, 2805 (2017).

51. Béa, $\mathrm{H}$. et al. Evidence for room-temperature multiferroicity in a compound with a giant axial ratio. Phys. Rev. Lett. 102, 217603 (2009).

52. MacDougall, G. J. et al. Antiferromagnetic transitions in tetragonal-like $\mathrm{BiFeO}_{3}$. Phys. Rev. B 85, 100406 (2012).

53. Rivero, P. \& Cazorla, C. Revisiting the zero-temperature phase diagram of stoichiometric $\mathrm{SrCoO}_{3}$ with first-principles methods. Phys. Chem. Chem. Phys. 18, 30686 (2016).

54. Halcrow, M. A. Jahn-Teller distortions in transition metal compounds, and their importance in functional molecular and inorganic materials. Chem. Soc. Rev. 42, 1784 (2013).

55. Cazorla, C. \& Stengel, M. Electrostatic engineering of strained ferroelectric perovskites from first principles. Phys. Rev. B 92, 214108 (2015).

56. Kresse, G. \& Furthmüller, J. Efficient iterative schemes for ab initio total-energy calculations using a plane-wave basis set. Phys. Rev. B 54, 11169 (1996).

57. Perdew, J. P., Burke, K. \& Ernzerhof, M. Generalized gradient approximation made simple. Phys. Rev. Lett. 77, 3865 (1996).

58. Blöchl, P. E. Projector augmented-wave method. Phys. Rev. B 50, 17953 (1994).

59. Cazorla, C. \& Boronat, J. Simulation and understanding of atomic and molecular quantum crystals. Rev. Mod. Phys. 89, 035003 (2017).
60. Komsa, H.-P., Rantala, P. P. \& Pasquarello, A. Finite-size supercell correction schemes for charged defect calculations. Phys. Rev. B 86, 045112 (2012).

61. Perdew, J. P. et al. Restoring the density-gradient expansion for exchange in solids and surfaces. Phys. Rev. Lett. 100, 136406 (2008).

62. King-Smith, R. D. \& Vanderbilt, D. Theory of polarization of crystalline solids. Phys. Rev. B 47, 1651(R) (1993).

\section{ACKNOWLEDGEMENTS}

Computational resources and technical assistance were provided by the Australian Government and the Government of Western Australia through the National Computational Infrastructure $(\mathrm{NCl})$ and Magnus under the National Computational Merit Allocation Scheme and The Pawsey Supercomputing Centre.

\section{AUTHOR CONTRIBUTIONS}

C.C. conceived the study and planned the research. C.M. and C.C. performed the theoretical calculations. Results were discussed by C.M., D.C., and C.C. The paper was written by C.M., D.C., and C.C.

\section{COMPETING INTERESTS}

The authors declare no competing interests.

\section{ADDITIONAL INFORMATION}

Supplementary information is available for this paper at https://doi.org/10.1038/ s41524-020-0344-3.

Correspondence and requests for materials should be addressed to C.C.

Reprints and permission information is available at http://www.nature.com/ reprints

Publisher's note Springer Nature remains neutral with regard to jurisdictional claims in published maps and institutional affiliations. Attribution 4.0 . adaptation, distribution and reproduction in any medium or format, as long as you give appropriate credit to the original author(s) and the source, provide a link to the Creative Commons license, and indicate if changes were made. The images or other third party material in this article are included in the article's Creative Commons license, unless indicated otherwise in a credit line to the material. If material is not included in the article's Creative Commons license and your intended use is not permitted by statutory regulation or exceeds the permitted use, you will need to obtain permission directly from the copyright holder. To view a copy of this license, visit http://creativecommons. org/licenses/by/4.0/.

(c) The Author(s) 2020 